\title{
ENTRE SALTOS Y CAÍDAS: ANÁLISIS DE LOS PRIMEROS DIEZ AÑOS DE ENSEÑANZA DE LA PSICOLOGÍA A DISTANCIA EN MÉXICO
}

\author{
Omar Moreno Almazán \\ UNAM \\ México
}

\begin{abstract}
RESUMEN
El presente trabajo tiene como objetivo presentar un breve recuento histórico de uno de los sistemas de educación a distancia más importantes de México: el Sistema de Universidad Abierta y Educación a Distancia (SUAED) de la UNAM, en la carrera de Psicología; siendo uno de los primeros programas educativos basados totalmente en sistemas e-learning en todo el país. Hoy el SUAED Psicología es una de las carreras universitarias más grandes en todo el país, con una demanda que se ubica en los tres primeros lugares a nivel nacional y una matrícula superior a 2,800 alumnos. Este escrito tiene como propósito exponer las principales condiciones a nivel administrativo, tecnológico y académico que le han representado avances y atrasos que, al ser expuestos, pueden ser de utilidad a otros sistemas educativos en su desarrollo y aprender de la experiencia, de la universidad más importante del país y una de las más importantes de Latinoamérica.
\end{abstract}

Palabras Clave:

educación a distancia, enseñanza de la psicología, historia, educación superior.

\section{BETWEEN JUMPS AND FALLS: ANALYSIS OF THE FIRST TEN YEARS OF TEACHING DISTANCE PSYCHOLOGY IN MEXICO}

\begin{abstract}
This paper aims to present a brief historical account of one of the most important distance education systems in Mexico: The Open University System and Distance Education (SUAED) of the National Autonomous University of Mexico (UNAM), in the psychology career; Being one of the first educational programs based entirely on e-learning systems throughout the country. Currently, SUAED Psychology is one of the largest university degrees in the country, with a lawsuit contesting the top three at a national level and an enrollment of more than 2,800 students. This paper attempts to expose some of the main administrative, technological and academic situations that have represented advances and delays that, when exposed, can be useful to other educational systems in their development and learn from experience, the most important university in the country and one of the most important in Latin America.
\end{abstract}

Keywords:

distance education, teaching psychology, history, higher education.

Bitácora del Artículo:

| Recibido: 1 de Febrero de 2019 | Aceptado: 11 Abril de 2019 | Publicado en línea: Julio - Diciembre de 2019 | 
Psicología de ayer y hoy | Entre saltos y caidas: análisis de los primeros diez años... Moreno-Almazán

\title{
Autoría y Derechos de Propiedad Intelectual
}

\section{ENTRE SALTOS Y CAÍDAS: ANÁLISIS DE LOS PRIMEROS DIEZ AÑOS DE ENSEÑANZA DE LA PSICOLOGÍA A DISTANCIA EN MÉXICO}

\author{
Omar Moreno Almazán \\ UNAM \\ México
}

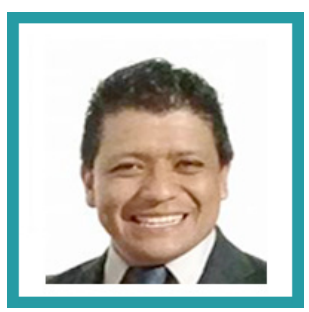

Omar Moreno Almazán

FES Iztacala, UNAM

Correo: almazanomar@gmail.com

Doctor en psicología educativa y del desarrollo por la UNAM. Fundador del SUAED Psicología en la UNAM. Actualmente es Presidente de la Academia de los Sistemas de Educación Presencial y a Distancia en Psicología y Miembro de la Sociedad Mexicana de Psicología desde 2001.

Ver más...

\section{CONTRIBUCIÓN DE LOS AUtORES}

Se atribuye al autor la responsabilidad única sobre el trabajar al concibir, desarrollar y coordinar el proyecto de investigación.

\section{AGRADECIMIENTOS}

Se agradece a todas las personas involucradas en la realización de esta investigación

\section{DAtos de Filiación de los Autores}

Sistema de Univeridad Abierta y Educación a Distancia, Facultad de Estudios Superiores Iztacala, UNAM.

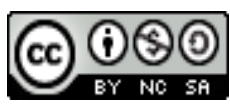

Copyright: (c) 2019 Moreno-Almazán, O.

Este es un artículo de acceso abierto distribuido bajo los términos de la licencia Creative Commons Reconocimiento-NoComercial 4.0 Internacional, por lo que su contenido gráfico y escrito se puede compartir, copiar y redistribuir total o parcialmente sin necesidad de permiso expreso de su autor con la única condición de que no se puede usar con fines directamente comerciales y los términos legales de cualquier trabajo derivado deben ser los mismos que se expresan en la presente declaración. La única condición es que se cite la fuente con referencia a la Revista Digital Internacional de Psicología y Ciencia Social y a su autor. 


\section{TABLA DE CONTENIDO}

EL INICIIO

El Sistema de Universidad Abierta y Educación a Distancia (SUAED), 34

Plan curricular del SUAED Psicología, 35

Gestión, coordinación y sedes, 35

Retos, logros y desafíos en la tecnología educativa, 37

Formación, servicio e investigación en Psicología, 38

Los siguientes saltos, 39

Una mirada al futuro del SUAED, 40
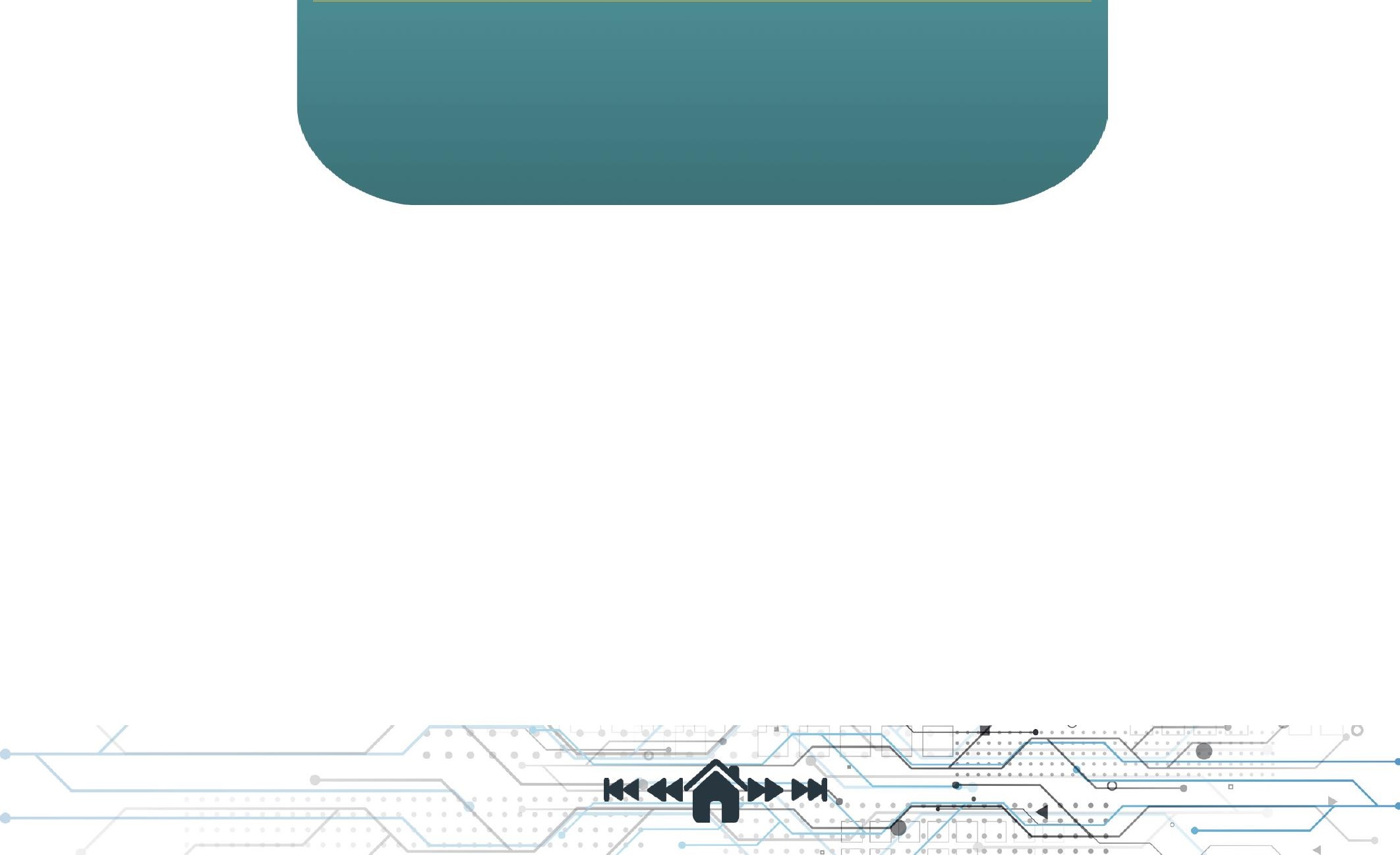
Psicología de ayer y hoy | Entre saltos y caidas: análisis de los primeros diez años... Moreno-Almazán

\section{EL INICIIO}

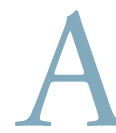

inicios de la década de los setenta del siglo XX, el rector de la Universidad Nacional Autónoma de México (UNAM), Pablo González Casanova (1970), impulsó un proyecto que formó parte de la reforma universitaria propuesta en su gestión y que consistía en crear un sistema alterno de enseñanza flexible e innovador en sus métodos de enseñanza y de evaluación, proponiendo así la creación del Sistema de Universidad Abierta (SUA), que tendría la distinción de no ser un sistema de enseñanza por correspondencia, una teleuniversidad o una universidad paralela, sino un sistema con métodos clásicos y modernos de enseñanza, apoyado en el diálogo, la cátedra, el seminario y el libro (UNAM, 1972).

El panorama que el rector exponía para sustentar esta propuesta visualizó el crecimiento considerable de una demanda de estudios superiores en la población, la cual se veía sujeta a una serie de prejuicios y que determinó como necesario el impulso de una reforma global en la UNAM. A ese respecto, decía que la solución a esta creciente demanda presentó nuevos retos al país y a la universidad; al país en el sentido de una necesidad apremiante de generar, en cantidades considerables, nuevas fuentes de trabajo altamente calificadas, para no sólo aumentar, sino mantener un ritmo de desarrollo económico, político y social; a la universidad, en una necesidad de aumento simultáneo de oferta y calidad en la enseñanza.

Respecto a este aumento en la calidad y oferta, representó cambios considerables en las estructuras educativas y la creación de la "Universidad abierta", totalmente flexible en la oferta de educación y la cual supondría nuevas formas de enseñanza, evitando gastos que corresponden a viejos conceptos y formas de trabajo de la Universidad, que resultan inadecuados ante las nuevas necesidades de su función educativa, y conservando y fortaleciendo sus objetivos de lograr un alto nivel científico, humanístico y tecnológico, para cumplir de modo eficaz su misión (UNAM, 1972)

Fue así que el 25 de febrero de 1972 el H. Consejo Universitario de la UNAM aprobó la creación del Sistema de Universidad Abierta (SUA), el cual tuvo como objetivos los siguientes aspectos.

- Ampliar los beneficios de la educación superior a mayores sectores de la población, por medio de métodos teórico-prácticos de transmisión y eva- luación de conocimientos mediante la creación de grupos de aprendizaje que trabajarán dentro o fuera de los planteles universitarios y centros de estudio.

- El desarrollo de formas que aseguren un alto nivel en la calidad del aprendizaje.

- La incorporación racional de los modernos medios electrónicos de comunicación educativa.

- Contribuir a fortalecer la eficiencia de los estudios universitarios.

Sus características primordiales son:

- Sistema de libre opción, para los alumnos y las facultades y escuelas de la Universidad.

- Se exigen los mismos requisitos de ingreso.

- La universidad otorga certificados, títulos y grados correspondientes al grado de estudio.

La estructura didáctica del SUA se proyectaría hacia tres procesos básicos (enseñanza, aprendizaje y comunicación) en los que intervienen el sujeto cognoscente, el objeto de conocimiento (saberes, prácticas, expresiones y manifestaciones) y la acción que los vincula y constituye. Su modelo educativo consideraría a los sujetos en diferentes figuras de estudiante y asesor, estableciéndose entre ellos una relación rica en aprendizaje (figura 1).

\section{Figura 1}

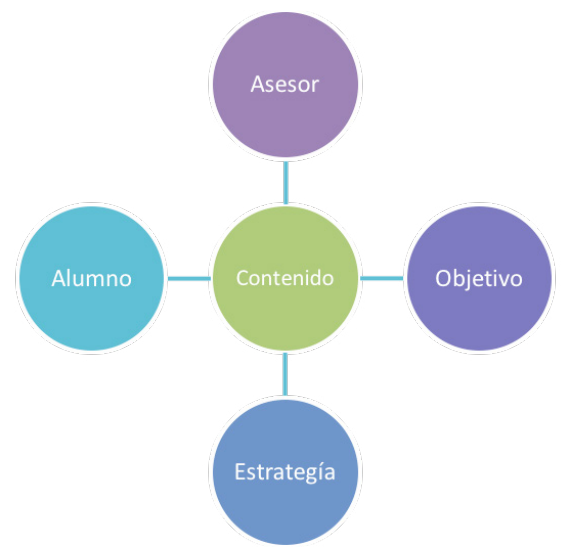

Modelo educativo del SUA. Fuente García Torres s/r

La relación de aprendizaje formaría e informaría a los sujetos, de acuerdo con sus orientaciones expresadas en el planteamiento curricular. Los sujetos con dos posibles roles intercambiables (de estudiante y asesor), tres funciones complementarias (enseñar, aprender y comunicar) y dos puestos fijos en la relación didáctica (docentes y alumnos) (García y Torres, s/f); de este modo, la relación didáctica establecida en este sistema ubica al alumno como agente central del proceso, donde los docentes se convierten en tutores que guían el proceso de autoaprendizaje y dotan de actividades y recursos a los alumnos; la comunicación, tanto sincrónica como 
asincrónica, se convierte en una base fundamental para el desarrollo de este proceso.

A partir de su apertura el sistema de educación abierta en la UNAM adquirió forma gradual. En sus primeros años las carreras que se abrieron en esta modalidad sólo consideraron algunas facultades, como: Facultad de Ciencias Políticas y Sociales, Contaduría y Administración, Derecho, Economía, Filosofía y Letras, Odontología y Psicología; sin embargo, su matrícula en los primeros años fue considerada a la par de la matrícula de los alumnos del sistema escolarizado (UNAM, 2005-2017).

Esta modalidad no representó la aparición de la enseñanza a distancia en México. La historia de la educación a distancia mexicana tiene orígenes que suelen ser discutidos, pero se tiene evidencia de algunos antecedentes en sistema de enseñanza donde no se consideró la presencialidad como un requisito básico. Autores como García-Aretio (1999) o Moreno (2015) hacen mención de casos como en el de la creación del Instituto Federal de Capacitación del Magisterio en 1947, el cual se enfocó en capacitación del profesorado en ejercicio profesional, o la creación de los Centros de Educación Básica para Adultos (antecedentes del INEA) en 1968. Sin embargo, un sistema que incorporó la tecnología educativa para crear una modalidad de enseñanza a distancia surgió en 1971 con la creación del sistema de telesecundarias, en el cual por medio de una serie de programas televisivos transmitidos vía satélite se generaba un proceso de enseñanza de educación media, sobre todo dirigido a poblaciones con alguna dificultad en su asistencia a clases regulares. Fue entonces que la década de los años setenta representó una revolución en los procesos de enseñanza abierto y a distancia, donde no sólo la UNAM establecio el SUA en 1972, sino que también otros sistemas como el modelo de Preparatoria Abierta, que surgió en 1973 en el Centro para el Estudio de Medios y Procedimientos Avanzados para la Educación (CEMPAE); el Sistema Abierto de Enseñanza en 1974 por parte del Instituto Politécnico Nacional; el Sistema de Educación Abierta en 1976, creada por el Colegio de Bachilleres; el Sistema Abierto de Educación Tecnológica Industrial, también en 1976.

Sin embargo, el concepto de educación a distancia comenzó en la década de los ochenta, donde los primeros intentos reconocidos se ubican en la Universidad Pedagógica Nacional (UPN), al crear en 1979 el Sistema de Educación a Distancia y en el Sistema de Enseñanza a Distancia de la Universidad Veracruzana en 1980, donde apoyados de tecnología generaron un sistema de enseñanza básicamente mixto.
Y no fue sino hasta la última década del siglo XX e inicios del XXI cuando las universidades incorporaron los sistemas basados en e-learning para crear programas educativos virtuales. Entre los más destacados se sitúan la Universidad Virtual creada por el Instituto Tecnológico y de Estudios Superiores de Monterrey (1997), el Sistema de Universidad Virtual de la Universidad de Guadalajara (2004), el Consorcio Clavijero en Veracruz (2005), la Universidad Virtual de Guanajuato (2007), la Universidad Virtual de Michoacán (2011), la Universidad Digital del Estado de México (2012) y la Universidad Abierta y a Distancia de México (UNAD), que se generó como un programa de la Secretaría de Educación Pública desde 2009. El surgimiento de estos sistemas enfrentaron retos en tres principales dimensiones: 1) el desarrollo de un sistema legal-administrativo que normalizara las operaciones con este esquema; 2) la aplicación de una didáctica que promoviera la interacción y el aprendizaje autorregulado, con una población que no había tenido que desarrollar tales habilidades en los sistemas educativos hasta ahora prevalecientes, y 3 la implementación de un sistema tecnológico que cubriera las necesidades y demandas de una naciente comunidad virtual.

En particular en el caso de la UNAM, donde se sitúa la enseñanza de la Psicología como profesión, bajo la modalidad e-learning, se ubica la incorporación de los sistemas a distancia a partir de una reorganización de las estructuras académicas en 1997. Fue entonces que el inicial sistema abierto (SUA) se enfocaría en convertirse en la Coordinación de Universidad Abierta y Educación a Distancia (CUAED) con dos propósitos: 1) redefinir las funciones de las direcciones y del consejo asesor, las normas, reglamentos y estatutos del SUA, y 2) desarrollar la base tecnológica requerida para crear programas de formación y capacitación del personal en entidades universitarias, apoyando de manera técnica, académica y con infraestructura. De este modo se fundó el Sistema de Universidad Abierta y Educación a Distancia (SUAED) en la UNAM, el cual proyectaría programas formativos a nivel de bachillerato, licenciatura, posgrados y educación continua. En 2005 el SUAED de la UNAM abriría licenciaturas basadas en la modalidad e-learning con un esquema basado en sedes territoriales donde la licenciatura en Psicología sería parte de la propuesta inicial, misma que en principio había sido propuesta por diversas facultades o entidades de la UNAM, pero que se quedaría con el programa creado por la entonces Jefatura de Psicología escolarizada de la Facultad de Estudios Superiores (FES) Iztacala, de la UNAM. Las implicaciones derivadas de esta decisión harían que la FES Iztacala creara un mecanismo de enseñanza de la psicología, que no se 
Psicología de ayer y hoy | Entre saltos y caidas: análisis de los primeros diez años... Moreno-Almazán

había visto antes en el país; y aun contando con algunos referentes, como en el caso de la Universidad Nacional de Educación a Distancia (UNED) de España, el caso de México sería distinto tanto por el modelo adquirido como la cultura educativa que nos distinguía; esto sin considerar las necesidades tecnológicas que pudieran responder a este reto.

\section{El Sistema de Universidad Abierta y Educación a Distancia (SUAED)}

Desde los sistemas de educación a distancia, la UNAM imparte la carrera de Psicología desde 2005, la cual se imparte desde la Facultad de Estudios Superiores (FES) Iztacala, con la inscripción en ese momento más de 2,800 alumnos que radican en todos los estados de la República y en otros países, sobre todo de Estados Unidos, Europa y Asia. El impacto de este sistema ha tenido una tendencia creciente.

Para el caso del sistema de Universidad Abierta se tiene un registro de su matrícula independiente a partir de la década de los años noventa; para el caso de la carrera de Psicología, es necesario indicar que su enseñanza en sistema abierto siempre ha sido desarrollada por la Facultad de Psicología, localizada en el campus central de la UNAM en la Ciudad de México; mientras que la enseñanza de la carrera de Psicología en el sistema a distancia ha sido impartido por la Facultad de Estudios Superiores de Iztacala, en el Estado de México. En la figura 2 se muestra el índice de matriculación del SUAED Psicología de la FES Iztacala.

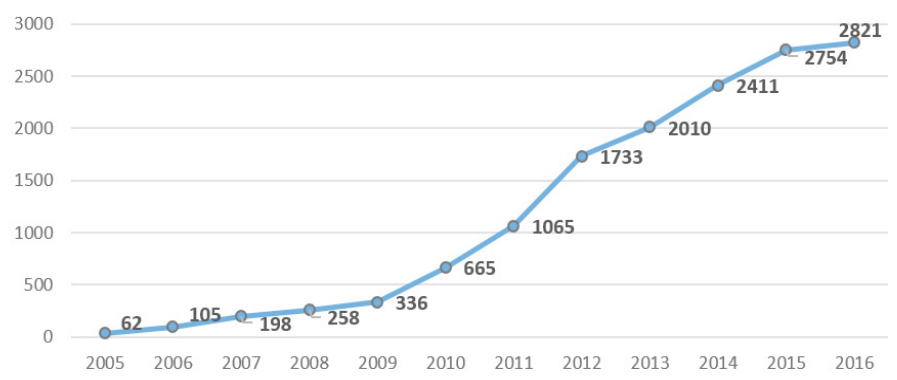

Figura 2

Matrícula del SUAED Psicología. Fuente: Agenda Estadística UNAM

En 2005 se inició la carrera con 62 alumnos. El sistema nació con el esquema de sedes, que consiste en el hecho de que la UNAM generó convenios con diversas instituciones para expandir su oferta educativa a sitios externos a la Ciudad de México. La primera sede se ubicó en el Estado de Tlaxcala, donde esa primera generación de 62 alumnos era casi en su totalidad de dicho estado. Después se incorporaron nuevas sedes y la matrícula se incrementó de modo acelerado, sobre todo ante la iniciativa de la rectoría de la UNAM entre 2010 a 2014 de ofertar lugares para las personas que no lograron obtener sitio en el sistema escolarizado. El sistema ha generado cambios que explicarían su tendencia al paso de los 10 primeros años, y que van desde su política y gestión por sedes, sus aplicaciones tecnológicas, su proceso pedagógico y de investigación, y hasta su egreso.

\section{Plan curricular del SUAED Psicología}

La planificación curricular tuvo el fundamento de sustentar sus objetivos generales institucionales y los de la licenciatura en Psicología en una variedad de supuestos básicos y metacurriculares que permitieran la actualidad en la formación de psicólogos que atendieran los problemas socialmente relevantes. De este modo se elaboró un diseño de plataforma curricular que pudiera incluir un tronco común con determinados objetivos formativos y después una sección formativa que fuera opcional para el alumno y que le permitiera profundizar respecto a un área de conocimiento.

Esta plataforma única de conocimientos básicos es un espacio curricular en el cual el alumno incursionará en los principios básicos relacionados con la manera en que se estructura el mundo empírico y el modo en que se organiza el mundo conceptual de la psicología, así como en los principios mediante los cuales se deriva el conocimiento de la psicología. A partir de esta situación se busca que la organización del plan de estudios sea consistente con la manera en que las teorías, los modelos psicológicos y el campo de servicio e investigación se relacionan entre sí en sus ámbitos teórico, metodológico y aplicado.

Con base en esto, el diseño de la Plataforma Única de Conocimientos Básicos contempla la inclusión, en el primer semestre, de la presentación de campo teórico de la psicología; en el segundo semestre el campo metodológico; en el tercero el campo aplicado, y en el cuarto semestre de esa zona de experiencia curricular una propedéutica con la que se pretende que el alumno adquiera una visión más amplia de las áreas de ejercicio profesional y de generación de conocimiento de la psicología que le permitan decidir en cuál de ellas incursionará en la siguiente gran sección formativa que le ofrece el plan de estudios que corresponde a la zona de profundización (figura 3). 


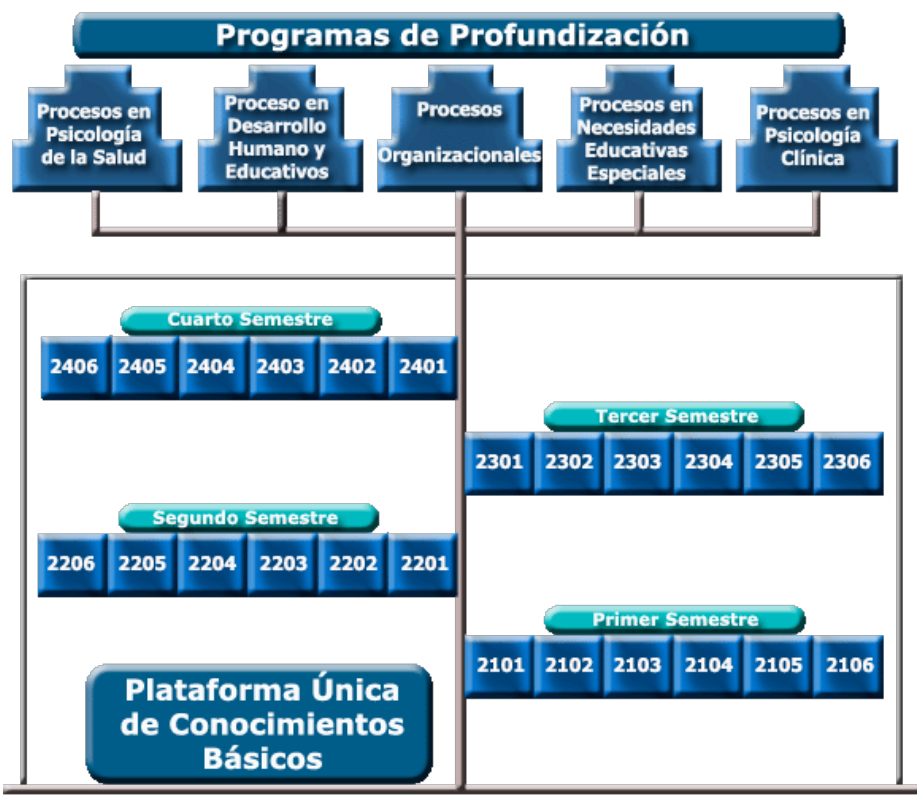

Figura 3

Mapa curricular de la Licenciatura en Psicología del SUAED

La estructura curricular tiene una base modular, es decir, tiene una serie de asignaturas regidas por los principios mencionados y que conforman tanto el tronco común como la zona de profundización. Dichos módulos son obligatorios para poder ingresar al área de profundización y el alumno tiene la libertad de elegir por semestre el número de módulos que juzgue convenientes a sus intereses. Los módulos que son parte del tronco común son: 1) antecedentes de la psicología; 2) psicología científica y sistemas teóricos; 3) psicología educativa; 4) psicología de la salud; 5) filosofía en psicología, y 6) neurociencias del comportamiento. Dichos módulos tienen sus propias formas de evaluación.

\section{Gestión, coordinación y sedes}

La idea inicial consistió en crear convenios entre la UNAM y algunas instancias externas para cubrir lo expuesto en el plan educativo nacional vigente de ese momento, en el cual se establecía que los programas educativos nacionales debieran seguir algunas líneas estratégicas que perfilaran una noción nueva de gestión educativa, de entre las cuales destacaron las siguientes.

- Avanzar hacia la equidad de la educación.

- Proporcionar una educación adecuada a las necesidades de todos los mexicanos.

- Impulsar el federalismo educativo, la gestión institucional y la participación social en la educación (Programa Nacional de Educación 2001-2006).

Dichos planteamientos llevan a la transformación de las prácticas y los procesos en la escuela, por lo que fue necesario considerar a la comunidad universitaria en su conjunto. El proceso que en ese momento surgió para la implementación del sistema fue mediante el establecimiento de los convenios para crear sedes, en donde la UNAM proporcionaría los programas curriculares, la gestión, la plataforma y a los docentes, mientras que las sedes ofrecerían el espacio físico, los recursos operativos inmediatos, los mecanismos de difusión y atención a alumnos. Además, cada parte involucrada en el convenio debía invertir recursos tecnológicos y monetarios en el proceso. Las sedes se enlistan a continuación.

- Tlaxcala 2005; mediante el Centro de Alta Tecnología y Educación a Distancia (CATED); fue la primera sede del convenio entre el gobierno de Tlaxcala y la UNAM; se ubicó en un inmueble histórico y la idea había sido crear un centro de desarrollo tecnológico educativo de vanguardia. El convenio no prosperó y casi 10 años después se perdió el inmueble y el CATED quedó reducido a unas cuantas oficinas pequeñas. La primera generación de Psicología surgió en dicha sede.

- Oaxaca 2006; por medio del Centro de Educación Continua, Abierta y a Distancia (CECAD) fue la segunda sede, la cual se distinguió por tener un proceso de gestión y de comunicación más eficiente que la primera sede. El convenio fue entre la UNAM y la Universidad Autónoma Benito Juárez de Oaxaca (UABJO); sin embargo, el convenio también se fue perdiendo y la UABJO mantiene la sede casi ya de manera independiente a la UNAM.

- Hidalgo 2007; fue un convenio entre la UNAM y el gobierno del estado de Hidalgo, donde se establecieron dos sedes: 1) al norte del estado, en San Felipe Orizatlán, y al sur en Tlaxcoapan. Su dinámica fue muy eficiente en los primeros años, llevando sistemas de educación a sitios remotos y lejanos a las ciudades principales. El convenio se rompió casi cinco años después; desparecieron por completo las actividades y difusión en dicha entidad.

- Chimalhuacán 2008; mediante un convenio entre la presidencia municipal de Chimalhuacán (Estado de México) y la UNAM se estableció un centro educativo que tuvo un acelerado crecimiento en infraestructura y versatilidad educativa, fundando el Centro Universitario Chimalhuacán $(\mathrm{CUCH})$ con una identidad muy particular. Aunque no se ha declarado el quiebre de dicho convenio, la UNAM dejó de modo gradual las actividades en dicha sede, la cual se ha mantenido de manera autónoma. Es necesario indicar que, en el caso de la carrera de Psicología, se asignó la primera generación en 
Psicología de ayer y hoy | Entre saltos y caidas: análisis de los primeros diez años... Moreno-Almazán

agosto de ese año, pero ante la posibilidad de que la FES Iztacala no fuera designada en el convenio para su atención, los alumnos de dicha generación no iniciaron actividades académicas sino hasta noviembre de ese año, donde la FES Iztacala fue designada.

- Toluca y Ecatepec (Estado de México) 2010. Fueron sedes que se establecieron mediante el convenio entre el gobierno del Estado de México y la UNAM. La intención fue incorporar a la UNAM en el programa de Universidad Digital y ampliar la oferta educativa de la entidad; sin embargo, el convenio no prospera porque ambas partes adquieren trayectos opuestos y las sedes se convierten en centros de captación de alumnos.

- Puebla 2012; fue creada por un convenio entre la Universidad del Desarrollo del Estado de Puebla y la UNAM; se generó la sede con la intención de ampliar la oferta educativa a la zona del Golfo. Hasta ahora el convenio se mantiene al menos en sus actividades básicas, aunque la UNAM se encarga de todos los procesos académicos y administrativos.

Actualmente la carrera de Psicología a distancia es gestionada por la UNAM desde su campus Iztacala, la cual se encarga de los procesos académicos, tecnológicos y administrativos; por su parte, la CUAED funge como una instancia de gestión, aunque en un aparente aislamiento o autonomía para las facultades. La estructura coordinadora de la carrera de Psicología tiene una coordinación central (semejante a una jefatura de carrera) que es designada a la persona que funge como jefe de tal carrera y quien deberá coordinar todos los trabajos relacionados a su sostenimiento y desarrollo. De esta coordinación se desprenden las siguientes áreas.

1. Desarrollo Académico y Extensión. Oficina encargada de todos los procesos de contratación, asignación de horas, establecimiento de grupos, aspectos relativos a los recursos humanos docentes, cursos, diplomados y actividades de actualización.

2. Departamento de Desarrollo Tecnológico. Área encargada de la organización, actualización y mantenimiento de la plataforma virtual, los sistemas en red y todos los aspectos tecnológicos e informáticos.

3. Asuntos Estudiantiles. Área encargada de la atención de todos los procesos estudiantiles, desde su ingreso hasta su titulación; cubre aspectos como difusión y becas, programas de tutorías, vinculación con sedes, prácticas profesionales y servicio social.

4. Desarrollo de Proyectos Educativos. En esta área se elaboran actividades relativas a la actualización, el diseño instruccional, así como procesos de segui- miento, actualización y/o modificación de los planes de estudio. A su vez, se enfoca en los trabajos de los cuerpos colegiados pertenecientes a la carrera.

\section{Retos, logros y desafíos en la tecnología educativa} EI SUAED inició actividades académicas en febrero de 2005 con 62 alumnos adscritos a la sede Tlaxcala. Su atención tuvo dos principales fuentes de contacto tecnológico: 1) el uso de correo electrónico institucional, proporcionado por la FES Iztacala, y 2) el uso de una plataforma desarrollada por la propia facultad, denominada Campus Universitario de Aprendizaje en Línea de Iztacala (CUALI), en la cual fueron matriculados los alumnos y los 12 tutores-docentes iniciales; dicha plataforma estaba hospedada en servidores en la misma facultad, creada y basada en sistemas operativos Solaris y Linux. El sistema CUALI operó con lenguajes como Perl, con la cual se desarrollaron las primeras actividades, además del uso de HTML, Flash y Java. La plataforma tecnológica CUALI operó durante un año y medio porque se trabajó en la creación de nuevos sistemas que fueran lo suficientemente robustos para la matriculación a mayor escala que se tendría contemplada a futuro (Silva-Rodríguez, 2015). En esa época la Dirección de Sistemas y Telecomunicación de la UNAM (DGTIC) asignó a los tutores una clave de acceso a internet por modem alámbrico como servicio de la universidad, donde era necesario desconectar la línea telefónica para acceder a internet.

En 2006 se comenzó la creación del Sistema de Educación Abierta y a Distancia (SEAD), que inició operaciones en 2007, brindando la posibilidad de que tuviera más funciones de organización, asignación de perfiles, mecanismos de comunicación e información, así como la diversificación de actividades formativas. Trabajó con el sistema operativo Fedora y los mismos lenguajes que CUALI. Para entonces, Silva-Rodríguez (2015) indica que la intención de la FES Iztacala era convertirse en un lugar de vanguardia tecnológica, para lo cual no se escatimaron recursos para la creación de estas plataformas, donde la dirección de la FES Iztacala de esa época invirtió más de 25,000 dólares en el SEAD, la cual no soportó su permanencia, presentando bloqueos, caídas de su sistema y ausencias de tiempo muy considerables. Debido a ello, SEAD operó sólo un año, siendo sustituida en 2008.

Hacia principios de 2008 surgió como respuesta emergente el Sitio de Apoyo Educativo (SAE) como principal medio de comunicación que permitió a los tutores el envío masivo de mensajes con instrucciones y fechas de publicación de trabajos, así como recomendaciones 
de trabajo; también estableció la comunicación individual monodireccional tutor-alumno, generando vías de comunicación de manera individual en avances particulares, notificaciones de retardo en las publicaciones, sugerencias personales para potenciar su desempeño académico y motivar al alumno a proseguir o mejorar su trabajo. Dentro de sus funciones introductorias están los cursos introductorios diseñados por el asesor, los cuales proporcionan un panorama general de los contenidos teóricos, lo que permite al alumno introducirse a la temática, porque dicho curso está redactado como una exposición de los contenidos, siendo apoyada dicha exposición por materiales interactivos situados en los pizarrones electrónicos (Moreno, 2008).

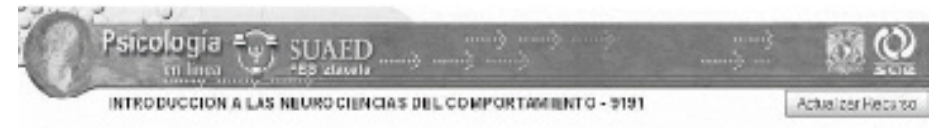

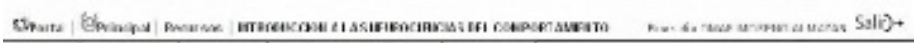

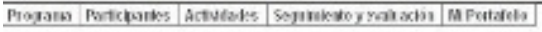

\section{La Historia Reciente de las Neuroclencias del Comportamiento}

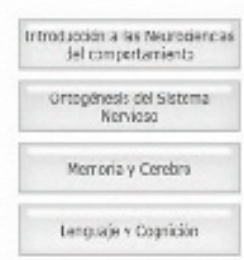

Figura 4

Entorno gráfico de un módulo del SAE, 2008

SAE fue la primera plataforma que operó con un núcleo basado en Moodle 1.7 (figura 4); sin embargo, tuvo elementos importantes que le hicieron perder su utilidad: La FES no tenía el código original del sistema, por lo que no podía hacerle modificaciones; estaba hospedada en servidores que fueron perdiendo potencia ante la cantidad de alumnos e interacciones que se hacían, cayéndose durante periodos que podían durar unos cuantos minutos y hasta más de cuatro días; operó durante cinco años sin posibilidad de ser actualizada, lo cual representó una importante limitación en los procesos de interactividad para un sistema e-learning; aunado a eso, por iniciativa de la coordinación de esa época, se estipuló que el SAE era el único espacio institucional para impartir docencia, y quien no entrara a dicha plataforma era equivalente a no asistir a clases (Oficio FESI/SCT/2983/11 del Consejo Técnico de la FES Iztacala). Esto último representó graves problemas en la normatividad y estabilidad del SUAED, lo que desató inconformidad en la planta docente, la creación de grupos reaccionarios de alumnos y la aparición de iniciativas tecnológicas independientes que dieron respuesta a esta limitación, aun con las problemáticas laborales que esto implicó.
A partir de 2014 se trabajó en una nueva propuesta tecnológica, la cual también estaba basada en Moodle, en una versión 2.4, y la cual tuvo con muchos más permisos, actualizaciones y estabilidad; fue entonces que en la segunda mitad de ese año se inauguró la nueva plataforma denominada Iztacala en Red (IRED). Dicha plataforma tuvo una serie de características en su núcleo central que ninguna otra plataforma había tenido: capacidad de actualización, módulos de blog, de encuesta y de exámenes en línea, sistema de bloques, de antivirus ClamAV, de estadísticas y de matriculación externa. Incluso otra novedad fue que IRED se hospedó en un servicio particular en la nube, siendo uno de los primeros sistemas de toda la UNAM en manejarse en esa manera, lo cual atrajo ventajas y vanguardia a la institución; sin embargo, por políticas derivadas de la dirección de la facultad, hubo la obligación de retirar la plataforma de la nube y hospedarla en servidores universitarios; no obstante, dadas las múltiples capacidades de la plataforma y de sus administradores, su funcionamiento se ha mantenido vigente. Con la aparición de IRED fue posible mantener una plataforma que podría estar en línea las 24 horas, los 365 días del año, lo cual representó una innovación que no se había tenido en Iztacala en ningún otro momento. Hoy aún se utiliza dicha plataforma en una versión Moodle 3.2 (figura 5), la cual cuenta además con una versión para dispositivos móviles.

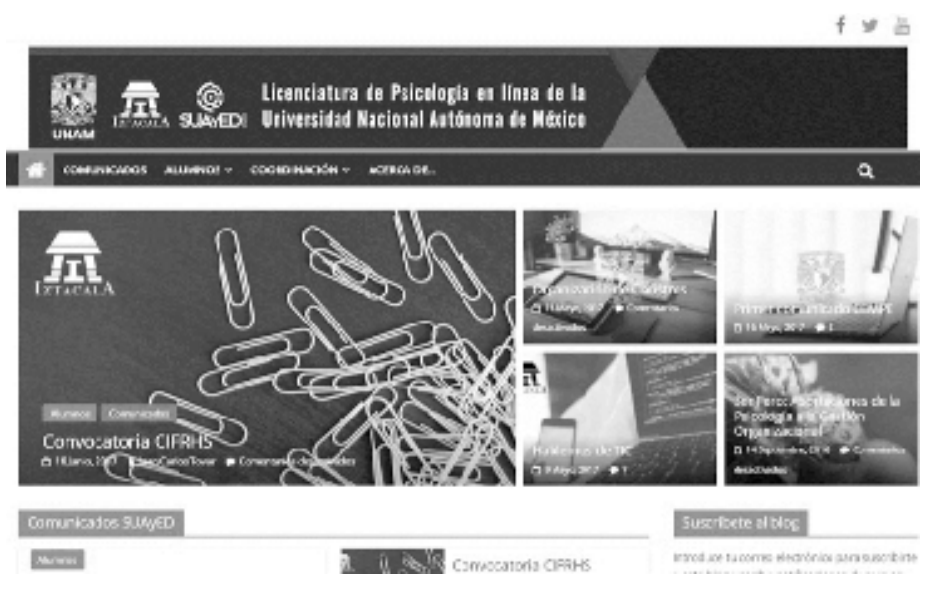

Figura 5

Portal de inicio de IRED 2017

Durante el desarrollo del sistema a distancia, otras aplicaciones y objetos han sido utilizados. El sistema inició utilizó como dinámica principal el uso de foros y envío de trabajos escritos en Word, su mayor forma de aplicación. El correo electrónico institucional era el único mecanismo de interacción, siendo en ocasiones expandido hacia el uso de videoconferencias entre las sedes y el campus central, requiriendo con ello la comunicación 
Psicología de ayer y hoy | Entre saltos y caidas: análisis de los primeros diez años... Moreno-Almazán

en tiempo real y la asistencia de los alumnos a las sedes. Con la entrada de Moodle mediante la plataforma $\mathrm{SAE}$, hubo una extensión en la comunicación por medio de un sistema de mensajería interna, además de la incursión de sistemas externos como el uso de Skype o de Streaming. Al contar con la plataforma IRED los sistemas de telecomunicación se expandieron hacia mecanismos de videoconferencia particular mediante sistemas como Hangouts, Skype, la inclusión de redes sociales, la creación de grupos de comunicación, además de emigrar hacia un sistema de correo institucional por medio del servicio de Gmail corporativo, lo cual ha permitido expandir los servicios de comunicación e interacción. Además la plataforma IRED hoy representa el centro principal de interacción y formación profesional.

Otros mecanismos institucionales se han utilizado como auxilio en los procesos académicos y administrativos, ajenos a las plataformas académicas. Entre los sistemas más destacables se encuentra el Sistema Integral de Control Escolar (SICE), el cual es un mecanismo que funciona como una base de datos y un sistema de procesos administrativos en toda la facultad; se trata de una aplicación creada de manera institucional. El Sistema de Exámenes en Línea de Iztacala (EXLI) fue durante mucho tiempo el sistema que se utilizó para el desarrollo de exámenes en línea en el SUAED, el cual consta de un sistema de hospedaje y distribución de reactivos por medio de códigos, el cual programaba la presentación de exámenes. Aunado todo ello al portal de la Facultad de Iztacala, que ha fungido como el medio de presentación oficial de nuestro plantel.

\section{Formación, servicio e investigación en Psicología}

Con el transcurrir de los años el SUAED se ha convertido de manera gradual hacia los sistemas de formación a distancia. En sus primeros años la presencia de los estudiantes en las sedes o en el campus central, así como la interacción en tiempo real con los docentes, era un requisito ineludible y de vanguardia para su época. Durante este periodo incluso se estilaron jornadas de difusión, atención y de intercambio académico, donde los docentes tutores asistían a las sedes en los distintos puntos del país. Es evidente que dicha estrategia facilitaba los procesos de adquisición de aprendizaje, pero excluía a quienes no podían estar presentes, dejando de lado los preceptos de la educación basada en e-learning. Los procesos formativos han adquirido una mayor independencia, basadas en algunas de las siguientes estrategias.

- El uso de mecanismos de tecnología educativa de la web 2.0 y 3.0 .
- La expansión de convenios y/o contactos que establece la UNAM con diversas instituciones locales de todo el país, con el objetivo de generar espacios de formación, servicio y práctica profesional de sus estudiantes.

- Ampliar las actividades formativas para los alumnos.

- Propiciar el desarrollo de prácticas profesionales supervisadas tanto por los docentes como por los responsables de las instituciones receptoras.

- Impulsar los procesos de investigación.

- El desarrollo y perfeccionamiento constante de estancias de servicio local y foráneo

De acuerdo con Rodríguez y Seda (2013), en educación superior se reconoce cada vez más la importancia de enfrentar al aprendiz a problemas reales propios de su profesión, es decir, a "situaciones donde el estudiante desarrolle las competencias propias de su carrera. En Psicología específicamente, existen prácticas formativas donde se asigna al estudiante un papel cercano a lo que será su vida laboral"; de este modo, en el caso de SUAED Psicología se ha puesto atención en la necesidad de crear un programa curricular que vaya desde aspectos teóricos e históricos, metodológicos y de conocimiento de las áreas de aplicación; después van hacia módulos propedéuticos que permiten a los alumnos conocer las diversas áreas de aplicación y elegir de entre cinco programas de especialización, la que ellos tengan más competencia e interés. La elección de las áreas de profundización o especialización se efectúa después culminar sus módulos propedéuticos (en 4o. semestre), y entonces eligen entre las áreas de psicología: clínica, educativa, de las necesidades educativas especiales, de la salud u organizacional. Durante su estancia en estas áreas, ellos desarrollan actividades prácticas profesionales en diversas instituciones o comunidades, así como su servicio social de 800 horas, investigaciones y finalizan con su proceso de titulación.

En los primeros años hubo procesos de investigación breves o locales, los cuales fueron difundidos en diversos escenarios científicos. Hoy existen iniciativas de profesionalización y de investigación que aprovechan el uso de tecnología educativa y la incorporación de proyectos de investigación, financiada o particular, que propicia la inclusión de alumnos, docentes, profesionales e investigadores, en actividades tanto de actualización, de investigación y/o de profesionalización. Entre los ejemplos más destacados están los proyectos como el del Centro de Atención Psicológica y Educativa (CAPED) a cargo de la doctora Anabel de la Rosa; el proyecto de Habilidades Lingüísticas y Orales para la lectura y escritura en niños preescolares a cargo de la doctora Esperan- 
za Guarneros; Promoción de la Salud Sexual en hombres a cargo del doctor Ricardo Sánchez; Estudio de Sistemas Complejos en Ambientes Virtuales de Aprendizaje con el doctor Alejandro Miranda; Comportamiento Sexual y prevención en jóvenes, con el doctor David Javier Enríquez, y el sistema de Actualización en Formación Profesional en Psicología en Sistemas Jurídicos con el doctor Omar M. Almazán, entre otros. Cada una de estas iniciativas ha propiciado el desarrollo de investigaciones y prácticas profesionales que permiten la inclusión situada de los estudiantes a escenarios reales y temas de actualización.

A partir de 2014 surgieron en el SUAED iniciativas internas que fortalecieron el enriquecimiento formativo de los estudiantes: la creación del Encuentro Anual de Estudiantes en Psicología, iniciativa que tuvo por intención el acercamiento de los alumnos a nivel presencial y virtual que permitiera el arraigo de su identidad y el intercambio de conocimiento derivado de sus proyectos de trabajo académico en sus distintas formas y entidades. El desarrollo del área de Extensión Universitaria que posibilitó la creación de cursos extracurriculares en línea, de actualización y de perfeccionamiento de conocimiento. El desarrollo de un mecanismo automatizado de actividad docente y de alumnado, con el propósito de detectar la ausencia de actividad de las personas, con el objetivo de proporcionar mecanismos de ayuda y superación. La implementación minuciosa de evaluación docente, con la intención de retroalimentar y motivar el desempeño de los tutores en su ejercicio formativo por medio de un sistema robotizado que se implementó en la plataforma.

Aunque en el periodo de 2015 a 2016 estas iniciativas presentaron una pasividad y no hubo desarrollo, la herencia dejada desde 2014 permitió que el SUAED mantuviera vigencia en sus procesos tecnológicos y formativos; hacia 2017 los procesos de difusión y formación retomaron rumbo con iniciativas nuevas, como la creación de cursos virtuales asincrónicos, la inclusión de redes sociales del SUAED y la emisión del Boletín Digital SUAED, que permite conocer diversas temáticas de interés tanto interno como externo, comunicación directa y mayor proceso de identidad universitaria.

Hasta este punto histórico, la constitución de un sistema de enseñanza de la Psicología, desde un sistema basado en el e-learning, había logrado caídas considerables en las dimensiones legales, administrativas, tecnológicas y didácticas; sin embargo, la creciente matrícula e índices de egreso, sus plataformas virtuales basadas en Moodle, la sistematización de sus mecanismos administrativos, la ampliación de su cobertura tanto a nivel nacional e internacional; así como la incorporación gradual de adita- mentos tecnológicos y didácticos para ampliar la dinámica formativa han representado los principales hitos de desarrollo que este sistema ha propiciado a la enseñanza de la Psicología nacional, así como de los mecanismos de educación virtual de nuestro país.

\section{Los siguientes saltos}

El nacimiento del SUAED Psicología se dio en un estigma de su escenario social e institucional marcado con algún escepticismo en las ideas de que la formación profesional en Psicología no podría hacerse por medio de sistemas de enseñanza en línea; para muchos académicos de gran trayecto en la FES Iztacala, la idea de crear el SUAED en una profesión que requiere la enseñanza de formas de intervención, de evaluación, de entrevista, dinámicas de grupo, etcétera, no tenía manera de hacerse mediante e-learning y pronosticaron que el SUAED fracasaría. Su escepticismo muchas veces ocasionó reacciones institucionales que salían del margen de la normatividad universitaria, como la prohibición de cualquier iniciativa de investigación en 2006 por parte de la Secretaría Académica o la limitación a un recurso tecnológico "oficial" que se tornó obsoleto en 2011.

Sin embargo el sistema comenzó a tener una mayor matrícula tras cada año, al grado de triplicar su capacidad de ingreso en un solo año y ampliar la oferta educativa. En este tenor, el SUAED enfrentó situaciones que a la larga fueron posicionando su condición. Cuando en 2013 la Dirección de la FES Iztacala expresa en público que "en el caso de Psicología SUAED, su problema radica en su baja tasa de egreso, ya que depende por completo del empeño de los estudiantes y de sus limitaciones de tiempo debido a sus compromisos laborales y familiares". Y remató diciendo: "[...] abiertamente expreso mi preocupación por el futuro de esta carrera; estamos en el proceso de diagnóstico para definir las acciones a seguir" (Dávila, 2013); era evidente que a pesar de tener varios años de existencia de la educación a distancia en el plantel, aún no se lograba entender su mayor esencia ni su particularidad multivariada, diversa y plural. El ejercicio estadístico que se haría consistió en comparar al SUAED con los indicadores de las licenciaturas presenciales (que además tenían más de 30 años de existencia). En ese momento la administración reportaba que mientras la carrera de Psicología del sistema presencial tenía una eficiencia terminal de $70 \%$ de toda su matrícula, junto con carreras como Medicina u Odontología, en el SUAED sólo era de $9.6 \%$.

No obstante, haciendo una verdadera comparación de los indicadores de titulación de la carrera de Psicología en los tres sistemas que tiene la UNAM, se puede

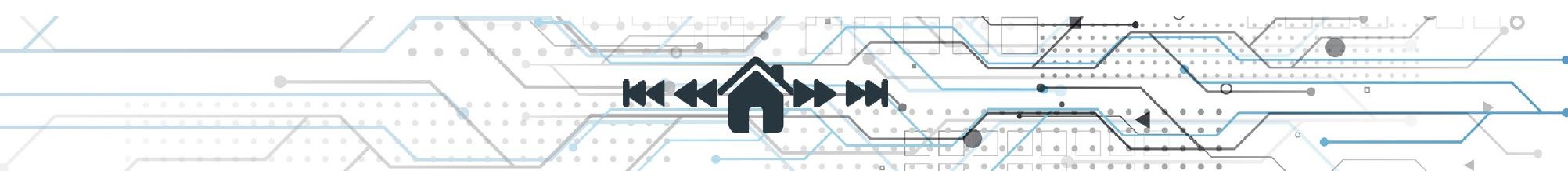


Psicología de ayer y hoy | Entre saltos y caidas: análisis de los primeros diez años... Moreno-Almazán

observar el crecimiento que han tenido dichas carreras. En la figura 6 se muestra el índice de egreso y culminación de estudios.

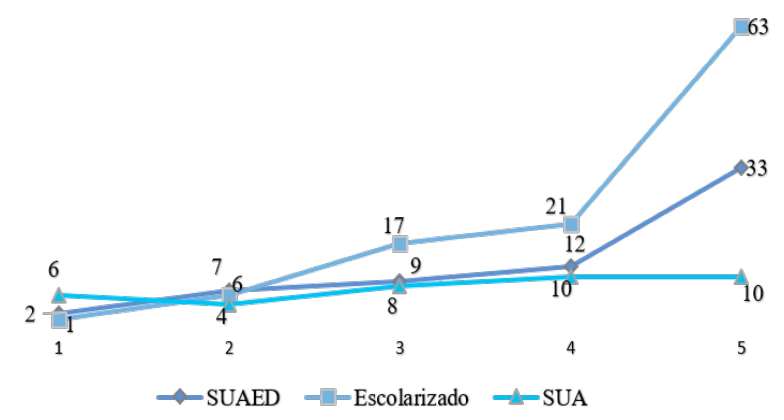

\section{Figura 6}

Tasa porcentual de culminación de estudios de Psicología en las tres modalidades en la UNAM. Fuente: Agenda Estadística UNAM

Se pueden apreciar la condición de culminación de estudios de la carrera de Psicología en sus cinco primeros años de existencia; para el caso del sistema escolarizado, corresponde a la carrera de Psicología de la FES (entonces ENEP) Iztacala, reportando los exámenes profesionales efectuados en sus cinco primeros años, a partir de 1979; en el caso del SUA correspondiente a la Facultad de Psicología y su titulación a partir de 1994. En el caso del SUAED se reportan sus primeras titulaciones a partir de 2010. El primer año en que cada carrera reportó esos datos, el sistema escolarizado sólo tituló a una persona, el SUA a seis y el SUAED a dos; después el crecimiento de estos indicadores tuvo un trayecto distinto, donde mientras en el sistema escolarizado hubo un crecimiento que, de manera explicable, es acelerado; los casos del SUA y del SUAED fueron un poco más lentos, siendo mayor en el SUAED que en el SUA. Esto permite observar que la implementación de un sistema de educación a distancia no es necesariamente desventajosa en relación con otras modalidades, y que los resultados formativos son equiparables a otros sistemas, con la ventaja adicional de que no es necesaria la presencialidad como en las otras modalidades.

\section{Una mirada al futuro del SUAED}

Se considera que los retos inmediatos que se esperan para el SUAED pudieran considerar los siguientes aspectos.

1. Actualización eficaz de su plan de estudios. Apegada a la experiencia profesional y a la investigación de vanguardia, no se podría concebir una aparente modificación a su plan curricular desde la ausencia de investigación, de experiencia profesional y práctica continua; tampoco podría verse la posibilidad de abrir el escenario curricular a todas las escuelas teóricas en la Psicología, sino más bien encauzarla al adiestramiento de conocimientos y habilidades en un solo enfoque teórico que pueda proporcionar demanda a las necesidades profesionales en cuanto a su intervención, formas de evaluación, procesos de prevención, mecanismos de enseñanza, y desarrollo y divulgación de la ciencia; todo enmarcado desde principios sociológicos y epistemológicos que den cabida a su adecuación desde un modelo curricular vigente.

2. Estabilidad contractual y normativa de los docentes. A más de 10 años de existencia de esta modalidad en la UNAM, aún no se tiene un marco legal que reconozca las figuras del docente en línea ni de sus características. En consecuencia, esto ha propiciado que se sigan los parámetros de contratación y desarrollo académico con los estándares que se utilizan para profesores de educación presencial.

3. Profesionalización de los docentes. Es necesario un plan que permita el manejo adecuado de los sistemas de tecnología educativa actual, de diseño instruccional y de estrategias didácticas y de autorregulación en línea.

4. Acelerar los procesos de mantenimiento y actualización de la plataforma y sistemas tecnológicos, de modo que se continúe la actualización del uso del Moodle, así como la implementación de las nuevas tendencias tecnológicas para la educación, que privilegien el desarrollo de un aprendizaje significativo, autorregulado, situado y correspondiente a la demanda social.

5. Integración estudiantil. Como consecuencia de iniciativas derivadas de la coordinación del SUAED en 2015 se eliminó como parte de los procesos formativos estudiantiles, su capacitación en el uso de paquetería estadística y de ofimática, así como la eliminación de actividades científicas. Por consiguiente, es necesario dotar a los estudiantes de éstas y otras habilidades que les permitan mantener un desarrollo competitivo y acorde a la demanda social.

6. Crear un programa de seguimiento de egresados y de vinculación con el sector laboral, ante el hecho de que no existe ningún mecanismo que permita conocer el desarrollo profesional de los alumnos egresados de este sistema ni de las condiciones y demandas que enfrentan en el campo profesional.

7. Potenciar la investigación en el SUAED, donde se promueva, reconozca, premie e incremente la participación de docentes y alumnos en actividades de investigación, relacionadas con el sector social y 
basadas en sistemas de aplicación comunitaria y de redes virtuales.

8. Sistematizar los procesos administrativos y de vinculación con el sector privado para que los estudiantes puedan desarrollar de manera más eficaz y efectiva sus procesos académicos y se contrarresten las acciones burocráticas.

Esta es una serie de estrategias necesarias para la ampliación y adecuación de un sistema e-learning; de cierto modo es imprescindible considerar tanto la realidad social y nacional, y las tendencias educativas mundiales que permitan observar un trayecto sólido hacia la formación profesional del futuro. De manera adicional e independiente a los distintos actores que suelen estar en los puestos de decisión y gestión de las instituciones educativas, es necesario destacar que la tendencia de un programa educativo depende en gran medida de las motivaciones y actuaciones de los involucrados directos, es decir, de los docentes y de los alumnos; sin estas dos figuras no es posible concebir un proyecto de escuela y del compromiso y profesionalismo de dichas figuras; se marcará la línea futura tanto del SUAED como de cualquier sistema de educación en cualquier lugar.

\section{Referencias}

Dávila, A. (2013). Primer informe de actividades. Pag. 36. Consultado el 07 de julio de 2017 en: http://direccion.iztacala.unam.mx/ PDFs/primer_informe_PDDA.pdf

González Casanova. (1970). Un gobierno universitario implica sobre todo el uso de la razón y el ejemplo de la conducta. Gaceta UNAM, vol. XIX, núm. 5 (15 de mayo, 1970), pp. 2-5. 39.

García-Aretio, L. (1999). Historia de la Educación a Distancia. RIED. Revista Iberoamericana de Educación a Distancia, volumen 2, No. 1, pp. 8-27

García, J. V., \& Torres, E. E. (s/f) Modelo Educativo del Sistema Universidad Abierta de la Universidad Nacional Autónoma de México. Manuscrito no publicado. CUAED:UNAM

Moreno, A. O. (2008). Sitio de Apoyo Escolar (SAE) como recurso de apoyo virtual de trabajo: La experiencia de psicología a distancia. Congreso Virtual Iberoamericano de Educación a Distancia

Moreno, M. (2015). La educación superior a distancia en México. Una propuesta para su análisis histórico. En Zubieta, J y Rama, C. (eds.) La educación a distancia en México: Una nueva realidad universitaria. (pp. 3 - 16). UNAM: México

Programa Nacional de Educación 2001 - 2006. Gobierno Federal. Recuperado el 6 de julio de 2017 desde: www.oei.es/historico/ quipu/mexico/Plan_educ_2001_2006.pdf

Rodríguez, F., \& Seda, I. (2013). El papel de la participación de estudiantes de Psicología en escenarios de práctica en el desarrollo de su identidad profesional. Perfiles Educativos. vol. XXXV, num. 140, pp. 82-99

Silva-Rodríguez, A. (2015). La educación a distancia en la UNAM. Una semblanza desde el SUAyED Psicología. Revista Digital Internacional de Psicología y Ciencia Social, 1(1), 1-422. doi:http://dx.doi.org/10.22402/j.rdipycs.unam.1.1.2015.30.1$\underline{422}$

Universidad Nacional Autónoma de México (1979 a 2016). Agenda Estadística UNAM. Desde 1979 y hasta 2016. Dirección General de Planeación. UNAM: México 
Psicología de ayer y hoy | Entre saltos y caidas: análisis de los primeros diez años... Moreno-Almazán

\section{Meta-Análisis del Artículo}




\section{Dimensión Cuantitativa}

\section{Perfil de Evaluación entre pares}
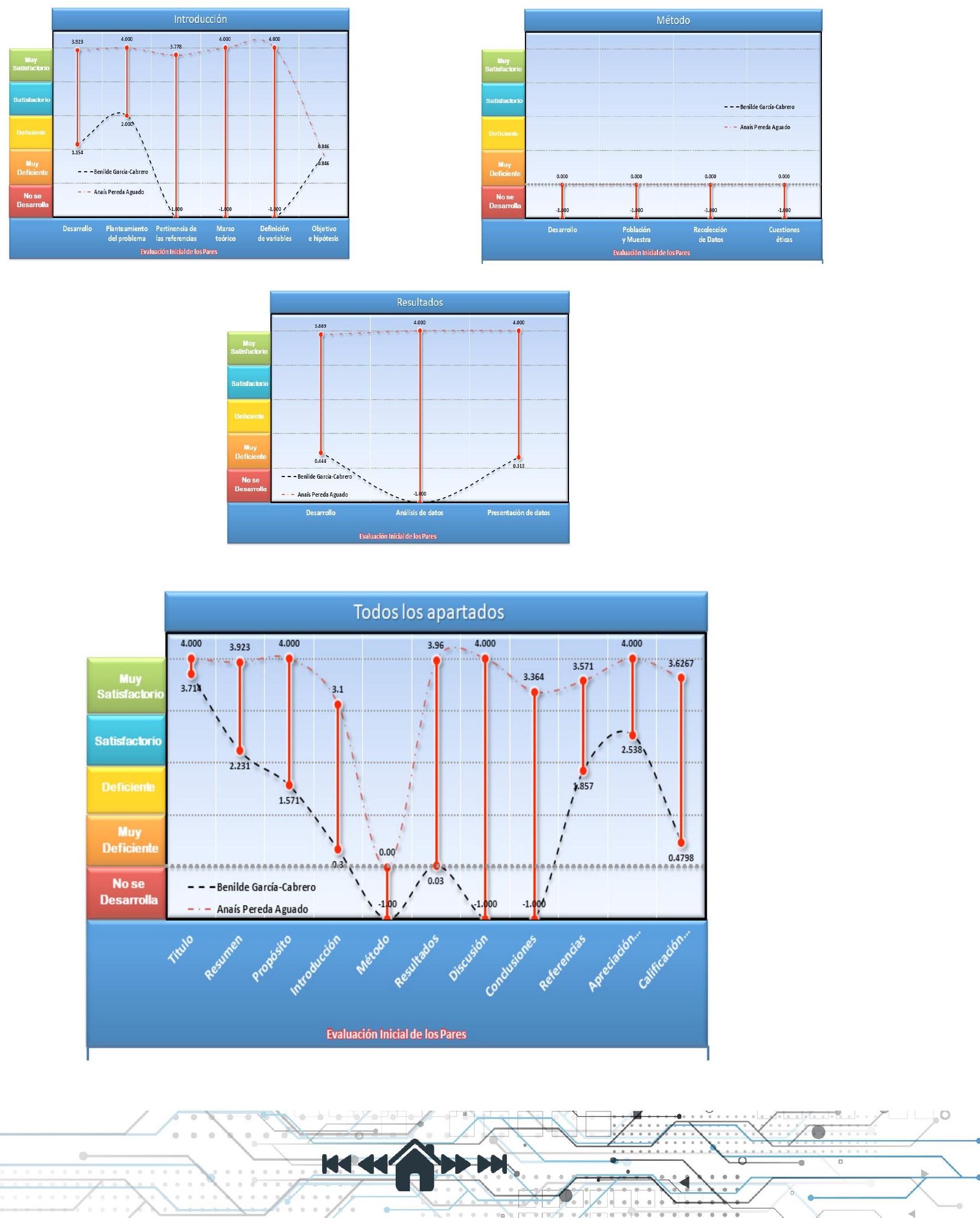
Psicología de ayer y hoy | Entre saltos y caidas: análisis de los primeros diez años... Moreno-Almazán

\section{Índice de Concordancia}

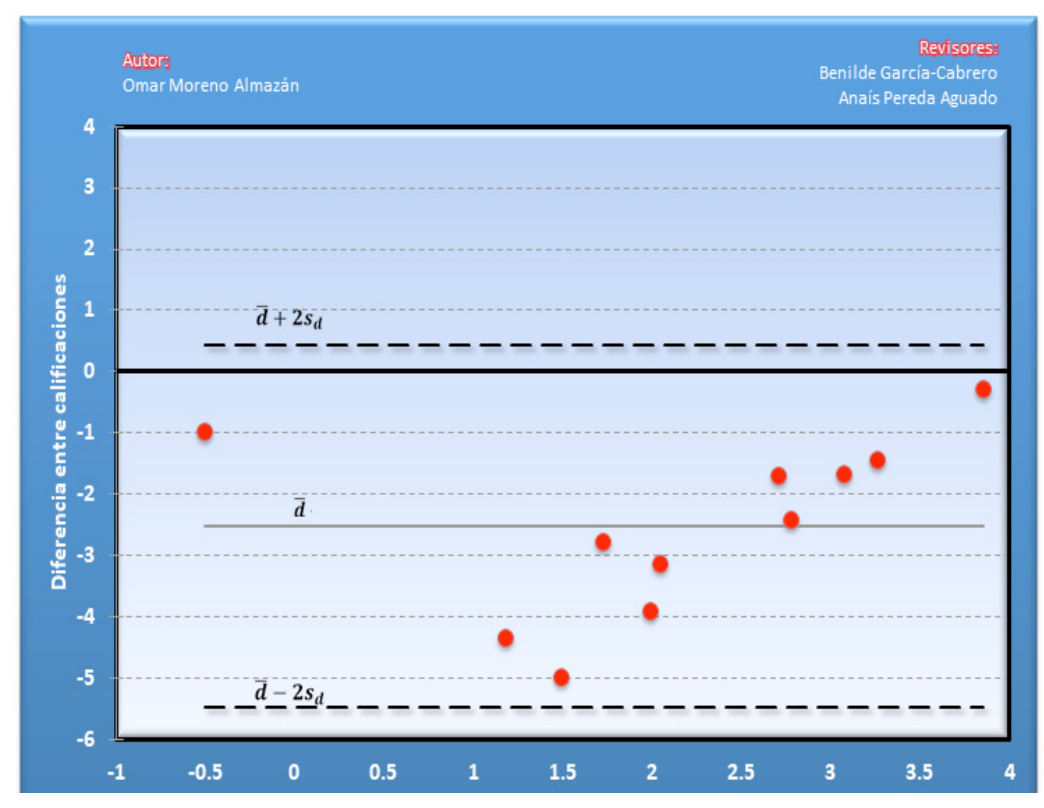

Índice de Acuerdo

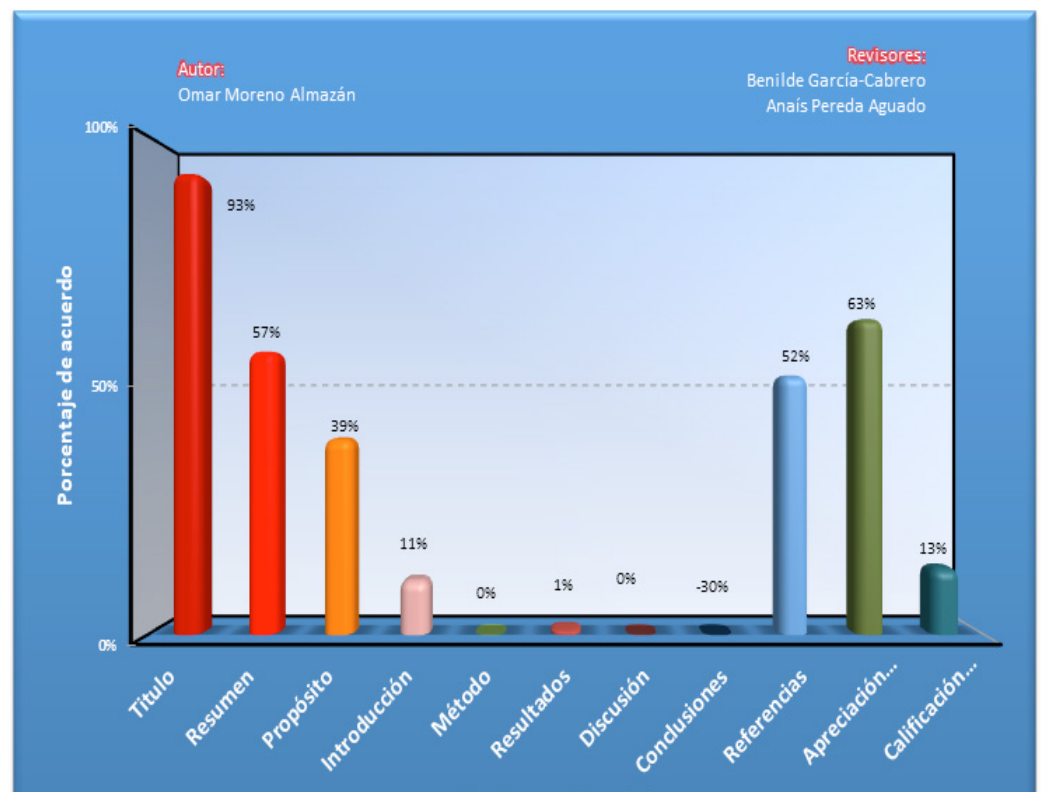




\begin{tabular}{|c|c|}
\hline Revisor 1 & Revisor 2 \\
\hline Benilde García Cabrero & Anaís Pereda Aguado \\
\hline
\end{tabular}

\section{Título/Autoría}

El título tiene dos partes, la primera resulta muy coloquial: entre saltos y caídas, y es necesario sustituirlo por una afirmación en la que el autor tome una postura más clara en la narrativa histórica que está planteando. El título debe reflejar una postura historiográfica, por ejemplo, la hermeneútica, mediante la cual el autor a través de la interpretación de textos hace explícito lo no evidente (interpreta la historia la historia y toma una postura respectoa ella). El título asignado da cuenta de que no hay una postura teórica sino una postura anecdótica en la que no existe un referente conceptual para narrar la historia de la enseñanza de la psicología a distancia en México.

\section{Resumen}

Se habla de recuento histórico pero cuál es el propósito de este recuento no queda claro "exponer algunas de las principales condiciones a nivel administrativo tecnológico y académico que le han representado avances y atrasos"... no está claro qué significa esto del recuento histórico y en ningún momento queda claro cuáles fueron los avances y cuáles fueron los atrasos.

\section{Ninguno}




\section{Revisor 1}

Revisor 2

\section{Próposito del Estudio}

El propósito debe estar vinculado estrechamente con el marco teórico y al método. Resulta interesante abordar la historia de la enseñanza de la psicología; sin embargo, no se tiene claridad al respecto de qué es lo que se pretende investigar, ya que "saltos y caídas" no son conceptos que permitan abordar el recuento histórico. Podría plantearse una postura hermenéutica de interpretación de textos, o bien un acercamiento a las representaciones que tienen los principales actores que Ninguno hicieron la historia de este proceso, en cuyo caso hubiese sido necesario entrevistarlos. Si el autor pretende realizar una interpretación histórica basada en textos, es necesario que adopte el enfoque hermeneútico y que lo analice sistemáticamente para poder abordar de manera sólida el objeto de estudio que propone. En vista de que no se plantean preguntas ni hipótesis, es difícil seguir la línea discursiva del trabajo presentado.

\section{Introducción}

No existe una introducción como tal, la historia de la enseñanza de la psicología a distancia se empieza a contar desde la primera página del documento sin haber planteado antecedentes introductorios, planteamiento del problema, preguntas de investigación, derivados de un marco teórico que justifique el estudio y el abordaje metodológico. Es necesario, primero recurrir a artículos seminales en el campo de la educación a distancia en general, analizando cuáles han sido los principales hitos históricos, los nudos problemáticos, las variables asociadas al crecimiento de la molaridad, los retos a los que se ha enfrentado y las soluciones que se han propuesto.

\section{Método}

Es necesario que exista una estructura más clara del documento en la que a partir de la introducción se establezcan categorías analíticas, con base en las cuales, seguido del planteamiento del problema, de la propues- Ninguna ta de método, se haga el recuento de la historia de la educación a distancia en general, y de la enseñanza de la psicología a distancia en particular. 


\section{Revisor 1}

Revisor 2

\section{Resultados}

No existe una sección bien definida de resultados y las gráficas y las figuras no ilustran claramente los datos que se presentan. Así mismo, es necesario que se ajusten al formato de la APA, considerando el uso de mayúsculas y curNinguna sivas tal como se señala en el manual. Así como la fuente, explicitando el año, en el caso de que exista.

\section{Discusión}

Se requiere que se desarrolle una sección específica para la discusión de los resultados encontrados, haciendo una síntesis de los mismos y contrastándolos con los Ninguna encontrados en otras investigaciones para finalmente acceder a la sección de conclusiones.

\section{Conclusiones}

Las conclusiones se deben resaltar los principales hitos históricos encontrados, los factores o variables que se vinculan con estos momentos críticos de la historia de la psicología a distancia en México, los actores que ges- Ninguna taron o fueron protagonistas de los principales acontecimientos así como las dificultades retos y oportunidades que se derivan del análisis historiográfico.

\section{Referencias}

En las referencias aparecen las siglas SUAyED y en todo el texto se cita como SUAED. El texto se basa solamente Ninguna en 11 referencias, lo cual resulta muy pobre. 
Psicología de ayer y hoy | Entre saltos y caidas: análisis de los primeros diez años... Moreno-Almazán 